\title{
Colesterolemia, Trigliceridemia e Excesso de Peso em Escolares de Santa Maria, RS, Brasil
}

\author{
Blood Lipids Abnormalities and Overweight Prevalence in Students \\ of Santa Maria, RS, Brazil
}

Cláudia Cruz Lunardi ${ }^{1}$

Cleci Menezes Moreira'

Daniela Lopes dos Santos ${ }^{1}$

1. Universidade Federal de Santa Maria, Santa Maria - Rio Grande do Sul

\section{Endereço para correspondência:}

Cláudia Cruz Lunardi

SMU / RCG/ QRS Rua I, casa $926-$

70630-200 - Brasília, DF

E-mail: claudia_lunardi@yahoo.com.br claudialunardi@gmail.com

\section{RESUMO}

Introdução: O nível de colesterol na infância é um preditor do nível de colesterol na vida adulta. As consequências do colesterol elevado, somadas a outros fatores de risco constituem problema mundial de saúde pública. Objetivos: Estimar a prevalência de hipercolesterolemia, hipertrigliceridemia e excesso de peso em estudantes do município de Santa Maria-RS. Métodos: Estudo transversal com 374 crianças de 10 a 12 anos de idade, de escolas das redes pública e privada, realizado no segundo semestre de 2005 na cidade de Santa Maria-RS. Foram determinados os níveis de colesterol total, triglicerídeos e estado nutricional. Resultados: As prevalências de hipercolesterolemia, hipertrigliceridemia e excesso de peso encontradas foram de 4,7\%, 8,9\% e 20,7\%, respectivamente. Não houve diferença significante entre sexo e rede pública e privada. As crianças com excesso de peso apresentaram maior prevalência de alterações lipídicas. Conclusões: A prevalência de alterações lipídicas pode ser considerada baixa, mas a prevalência de excesso de peso dos estudantes de Santa Maria mostrou-se relativamente alta, alertando à importância de políticas públicas e à necessidade de assistência pediátrica nessa faixa etária, visando o seu diagnóstico precoce e, principalmente, o aconselhamento nutricional e incentivo à prática esportiva, uma vez que as dislipidemias e o excesso de peso têm sido apontados como fatores de risco para as doenças cardiovasculares.

Palavras-chave: prevalência, colesterol, triglicerídeos, dislipidemia, excesso de peso, estudantes.

\section{ABSTRACT}

Introduction: Cholesterol level in childhood is a predictor of cholesterol level in adult life. The consequences of high cholesterol levels summed to other risk factors constitute a worldwide public health problem. Objective: To estimate the prevalence of blood lipid abnormalities and overweight among school students of the city of Santa Maria, RS, Brazil. Methods: A cross-sectional study with 374 children, aged between 10 and 12 years, from public and private schools of the city of Santa Maria, RS, conducted on the second semester of 2005. Total cholesterol levels (TC), fractions of cholesterol (HDL- C and LDL- C), triglycerides (TG) and nutritional status were determined. Results: The prevalence of hypercholesterolemia, hypertriglicemia and overweight found were 4.7\%, 8.9\% and 20.7\%, respectively. There was no significant difference between sexes or public and private schools. The overweight children presented higher prevalence of lipid alterations. Conclusions: The prevalence of lipid alterations may be considered low, but the prevalence of overweight among the students of Santa Maria was high, demonstrating hence the importance of public policies and the need for pediatric assistance at this age group. The aims here would be early diagnosis, especially for nutritional advising and stimulus for physical activities, since overweight and blood lipid abnormalities have been pointed out as risk factors for cardiovascular diseases.

Keywords: prevalence, cholesterol, triglycerides, dyslipidemia, overweight, students. 


\section{INTRODUÇÃO}

Diversos estudos têm demonstrado associação entre incidência de doenças cardiovasculares em adultos e fatores de risco desenvolvidos ou não controlados durante a infância(1-4). Dentre os fatores de risco encontram-se as dislipidemias, o hábito de fumar, a hipertensão arterial e a obesidade, que acometem cada vez mais crianças e adolescentes.

De acordo com o Committee on Nutrition ${ }^{(5)}$, as taxas de colesterol são influenciadas pela dieta e história familiar positiva para doenças. Recomendava-se a verificação da taxa de colesterol em crianças apenas quando se apresentava descendência de doença coronária na família; porém, atualmente, o Committee on Nutrition ${ }^{(5)}$ recomenda que seja verificada a taxa de colesterol das crianças sempre que possível, uma vez que hábitos alimentares influenciam nos níveis lipídicos. Especialistas recomendam que todas as pessoas conheçam seu nível de colesterol e façam um controle, no mínimo, a cada cinco anos, ou anualmente se o risco de doença cardíaca é elevado(1).

O nível de colesterol na infância é um preditor do nível de colesterol na vida adulta. Dados de vários países têm mostrado altos níveis de colesterol plasmático em crianças e adolescentes, levando muitos estudiosos a considerar a necessidade de prevenção pediátrica das doenças cardiovasculares ${ }^{(6)}$.

As consequências do colesterol elevado, somadas a outros fatores de risco, constituem problema mundial de saúde pública. Do ponto de vista clínico, a vigilância e a detecção precoce dos fatores de risco associados à hipercolesterolemia são fatores primordiais nas ações preventivas, com destaque para a obesidade infantil, cujo controle por meio da obtenção e manutenção do peso adequado para altura, sexo e idade, é factível em ações primárias de atenção à saúde ${ }^{(7)}$.

Neste sentido, este trabalho teve como objetivo verificar a prevalência de hipercolesterolemia, hipertrigliceridemia e excesso de peso em estudantes do município de Santa Maria-RS.

\section{MÉTODOS}

Esta pesquisa caracteriza-se como transversal descritiva. A coleta foi realizada no período de agosto a dezembro de 2005.

\section{População e amostra}

Foram realizadas as seguintes etapas, a fim de efetuar a seleção da amostra estratificada:

1) Cadastro das escolas e número total de escolares matriculados na $5^{\text {a }}$ série do ensino fundamental (correspondentes aos nascidos em 1994, equivalente à idade de 11 anos) no município de Santa MariaRS conforme rede de ensino, os quais foram obtidos por meio do senso escolar disponibilizados pela Secretaria Municipal e Regional de Educação.

2) Calculo amostral com grau de confiança de $95 \%$, margem de erro de 5\%, estratificados por rede de ensino e sexo.

3) Amostragem por conglomerados, por sorteio das escolas.

4) Contato com instituições de ensino a fim de explicar os objetivos do trabalho e verificar a disponibilidade das mesmas em participar.

5) Visita às escolas visando esclarecer aos escolares o trabalho, e encaminhar aos pais um documento explicativo sobre o trabalho contendo informações detalhadas e específicas sobre os benefícios da pesquisa, juntamente com autorização e Termo de Consentimento Livre e Esclarecido (TCLE) a serem assinados pelos responsáveis, condicionando a participação da criança de modo voluntário.

Depois de realizados os procedimentos de seleção amostral, descritos anteriormente, o cronograma para coleta de dados foi determinado. Coletaram-se os dados de todos os escolares que compareceram ao local com autorização e TCLE assinados pelos responsáveis.
Foram incluídos na pesquisa todos os estudantes nascidos no ano de 1994 e excluídos os que não respeitaram jejum de 12 horas (uma criança). Os avaliados que apresentaram alterações lipídicas receberam orientações quanto à importância do controle sérico e dos benefícios da alimentação saudável e das atividades físicas.

O estudo foi aprovado pelo Comitê de Ética em Pesquisa da UFSM (CAAE 0018.0.243.000-05).

\section{INSTRUMENTOS E PROCEDIMENTOS}

No momento da avaliação as crianças estavam descalças, vestindo shorts (ou calça) e camiseta. As medidas antropométricas foram determinadas por apenas um avaliador $(r=0,98)$ através da estatura (EST) e massa corporal (MC). As medidas de MC e EST seguiram o procedimento descrito por Alvarez e Pavan ${ }^{(8)}$. Para coleta dos dados foi utilizada uma balança, marca Plena, com resolução de 100g e um estadiômetro de metal, marca Cardiomed ${ }^{\circledR}$, com resolução de $1 \mathrm{~cm}$. A partir dos escores de MC e da EST, foi calculado o IMC a partir da seguinte equação: IMC $\left(\mathrm{kg} / \mathrm{m}^{-2}\right)$ $=\mathrm{MC}(\mathrm{kg}) / \mathrm{EST}^{2}(\mathrm{~m})$. Adotou-se os pontos de corte propostas pela Força Tarefa Internacional ${ }^{(9)}$ para classificar o estado nutricional das crianças.

A coleta sanguínea foi realizada por laboratorista do Laboratório de Análises Clínicas da Universidade Federal de Santa Maria (LAC/UFSM). Foram coletados $4 \mathrm{ml}$ de sangue por punção venosa a vácuo, após jejum de 12 horas, em frascos secos para dosagens bioquímicas. As análises foram feitas no LAC/UFSM pelo método colorimétrico-enzimático para todas as dosagens (CT e TG) utilizando o aparelho 917 Automatic Analyser Hitachi (Boehringer Mannhein).

Os pontos de corte utilizados para classificação dos níveis de colesterol foram os propostos pelas III Diretrizes Brasileiras sobre Dislipidemias(10), nas quais se têm valores desejáveis para CT $<170 \mathrm{mg} /$ dL e TG < 130 mg/dL; valores limítrofes para CT entre 170 e 199mg/dL; valores aumentados para CT > 200mg/dL e TG > 130mg/dL.

Os dados foram analisados pelo pacote estatístico Statistical Analysis System, versão 14.0, com intervalo de confiança de 95\% e nível de significância de 5\% ( $p \leq 0,05)$. Os dados apresentaram distribuição normal; desta forma, utilizou-se a análise descritiva, análise de variância Anova e teste do Qui-quadrado.

\section{RESULTADOS}

Foram avaliados 358 indivíduos (200 meninas e 158 meninos), sendo $76,2 \%$ de escolas públicas e 23,8\% de instituições privadas.

Ao analisar a tabela 1, verifica-se que a rede particular apresentou prevalências de alterações lipídicas superiores para $C T$, e a rede privada, prevalência superior para TG; no entanto, não foi observada diferença estatística entre as redes e sexo, nas três variáveis analisadas. $\mathrm{Na}$ amostra estudada, o excesso de peso (sobrepeso + obesidade) foi encontrado em 74 crianças (20,7\%), não sendo relatado nenhum caso de baixo peso.

Tabela 1. Dados descritivos e prevalência de alterações lipídicas e excesso de peso de acordo com o sexo. Santa Maria, Rio Grande do Sul.

\begin{tabular}{|c|c|c|c|c|c|c|c|}
\hline & & CT & $\%$ & TG & $\%$ & IMC & $\%$ \\
\hline \multirow{3}{*}{ Pública } & $F$ & $153,7 \pm 26,1$ & 5,5 & $87,1 \pm 41,6$ & 11,0 & $18,4 \pm 2,8$ & 15,2 \\
\hline & $M$ & $150,6 \pm 23,3$ & 1,6 & $69,7 \pm 32,5$ & 7,0 & $19,1 \pm 3,8$ & 27,3 \\
\hline & Total & $152,2 \pm 24,8$ & 3,7 & $78,9 \pm 38,5$ & 9,2 & $18,7 \pm 3,3$ & 20,9 \\
\hline \multirow{3}{*}{ Privada } & $F$ & $157,6 \pm 26,7$ & 7,3 & $85,5 \pm 49,4$ & 9,1 & $18,9 \pm 3,7$ & 21,9 \\
\hline & $M$ & $151,3 \pm 30,8$ & 10,0 & $73,6 \pm 38,4$ & 6, & $18,7 \pm 3,1$ & 16,7 \\
\hline & Total & $155,4 \pm 28,2$ & 8,2 & $81,3 \pm 46,0$ & 8,2 & $18,8 \pm 3,5$ & 20,0 \\
\hline \multicolumn{2}{|c|}{ Geral } & $153,0 \pm 25,6$ & 4,7 & $79,5 \pm 40,3$ & 8,9 & $18,7 \pm 3,4$ & 20,7 \\
\hline
\end{tabular}

Dados expressos em $\mathrm{mg} / \mathrm{dL}$ para variáveis lipídicas e $\mathrm{kg}^{-\mathrm{m}^{-2}}$ para IMC. $\%$ = Prevalência de alterações lipídicas. No caso do IMC, prevalência de excesso de peso (sobrepeso + obeso). 
Constata-se (tabela 2), percentual maior de meninas na faixa limítrofe e aumentado para o CT (20,9\% e 6,0\%, respectivamente) do que de meninos (17,2\% e 3,2\%, respectivamente). O oposto ocorre na variável IMC - há mais meninos com sobrepeso e obesidade do que meninas $(p>0,05)$.

Tabela 2. Prevalência de colesterolemia e excesso de peso de acordo com o sexo. Santa Maria, Rio Grande do Sul.

\begin{tabular}{c|c|c|c|c|c|c|c|c}
\hline \multirow{2}{*}{} & \multicolumn{4}{|c|}{ Colesterol total } & \multicolumn{4}{c}{ IMC } \\
\cline { 2 - 9 } & \multicolumn{2}{|c|}{ Limítrofe } & \multicolumn{2}{c|}{ Aumentado } & \multicolumn{2}{c}{ Sobrepeso } & \multicolumn{2}{c}{ Obesidade } \\
\cline { 2 - 9 } & $\mathbf{n}$ & $\%$ & $\mathbf{n}$ & $\%$ & $\mathbf{n}$ & $\%$ & $\mathbf{n}$ & $\%$ \\
\hline Feminino & 42 & 20,9 & 12 & 6,0 & 27 & 13,4 & 7 & 3,5 \\
\hline Masculino & 27 & 17,2 & 5 & 3,2 & 28 & 17,8 & 12 & 7,6 \\
\hline Geral & 69 & 19,3 & 17 & 4,7 & 55 & 15,4 & 19 & 5,3 \\
\hline
\end{tabular}

Ao analisar as escolas públicas e privadas (tabela 3), observou-se que, do total de 85 alunos das escolas privadas, 12 (14,1\%) apresentavam sobrepeso e cinco (5,9\%), obesidade. Dos 273 alunos das escolas públicas, foram encontrados $43(15,6 \%)$ e 14 (5,1\%) com sobrepeso e obesidade, respectivamente. As escolas públicas apresentaram percentual maior de estudantes na faixa limítrofe para CT e as particulares para CT aumentado.

Tabela 3. Prevalência de colesterolemia e estado nutricional de acordo com o rede de ensino. Santa Maria, Rio Grande do Sul.

\begin{tabular}{c|c|c|c|c|c|c|c|c}
\hline \multirow{2}{*}{} & \multicolumn{4}{|c|}{ Colesterol total } & \multicolumn{4}{c}{ IMC } \\
\cline { 2 - 9 } & \multicolumn{2}{|c|}{ Limítrofe } & \multicolumn{2}{c|}{ Aumentado } & \multicolumn{2}{c}{ Sobrepeso } & \multicolumn{2}{c}{ Obesidade } \\
\cline { 2 - 9 } & $\mathbf{n}$ & $\%$ & $\mathbf{n}$ & $\%$ & $\mathbf{n}$ & $\%$ & $\mathbf{n}$ & $\%$ \\
\hline Pública & 53 & 19,4 & 10 & 3,7 & 43 & 15,8 & 14 & 5,1 \\
\hline Privada & 16 & 18,8 & 7 & 8,2 & 12 & 14,1 & 5 & 5,9 \\
\hline Geral & 69 & 19,3 & 17 & 4,7 & 55 & 15,4 & 19 & 5,3 \\
\hline
\end{tabular}

Dentre as 74 crianças que apresentaram excesso de peso, 22 delas transitam na faixa limítrofe para a variável CT e oito já apresentam hipercolesterolemia (tabela 4).

Tabela 4. Prevalência de colesterolemia de acordo com o estado nutricional. Santa Maria, Rio Grande do Sul.

\begin{tabular}{c|c|c|c|c|c|c}
\hline \multirow{2}{*}{} & \multicolumn{6}{c}{ Colesterol total } \\
\cline { 2 - 7 } & \multicolumn{2}{|c|}{ Normal } & \multicolumn{2}{c}{ Limítrofe } & \multicolumn{2}{c}{ Aumentado } \\
\cline { 2 - 7 } & $\mathbf{n}$ & $\%$ & $\mathbf{n}$ & $\%$ & $\mathbf{n}$ & $\%$ \\
\hline Eutrófico & 228 & 80,3 & 47 & 16,5 & 9 & 3,2 \\
\hline Sobrepeso & 36 & 65,4 & 15 & 27,3 & 4 & 7,3 \\
\hline Obeso & 8 & 42,1 & 7 & 36,8 & 4 & 21,1 \\
\hline Total & 272 & 76 & 69 & 19,3 & 17 & 4,7 \\
\hline
\end{tabular}

\section{DISCUSSÃO}

Os dados relatados neste trabalho foram coletados apenas em um município da região central do estado do RS. Mesmo assim, esse estudo é importante, vista a precariedade de dados nacionais e, prin- cipalmente, regionais na literatura enfocando níveis de colesterol total, triglicerídeos e estado nutricional de crianças. Os resultados do presente estudo podem ter sofrido vieses de seleção, comprometendo assim sua generalização, uma vez que a seleção da amostra não foi feita de forma aleatória. Ressalta-se a importância da atenção e cuidado ao comparar os resultados com outros estudos, visto que os diversos autores trabalham com distintas faixas etárias, pontos de corte e outras características populacionais (raça/cor, crianças em geral e não necessariamente escolares).

Optou-se neste estudo pela classificação de Cole et al. ${ }^{(9)}$, uma vez que os autores utilizaram uma amostra brasileira para elaboração dos pontos de corte. Os valores sugeridos correspondem às referências para o IMC de 25 e $30 \mathrm{~kg} / \mathrm{m}^{2}$, adaptados para idade e sexo, para identificação de sobrepeso e obesidade, respectivamente. Consideraram-se como excesso de peso os indivíduos que apresentaram sobrepeso ou obesidade.

Os estudos encontrados, tanto em nível nacional como internacional, que tratam da prevalência de alterações lipídicas na faixa pediátrica, demonstram grande variabilidade nestes resultados. Em Campinas-SP(2), um trabalho realizado com 1.600 estudantes, com idade entre sete e 14 anos, verificou que 35\% da amostra apresentava hipercolesterolemia. Em Bento Gonçalves ${ }^{(4)}$, a prevalência encontrada foi de 27,9\%. Já no município de Itajaí-SC ${ }^{(11)}$ e Campina Grande-PB(12), a percentagem de crianças/adolescentes com CT aumentado foi de apenas 3,1\% e 3,3\%, respectivamente. Em relação às pesquisas internacionais, verificou-se que nos Estados Unidos ${ }^{(13)}$ a prevalência de hipercolesterolemia alcança percentagens de $9,8 \%$ a $16 \%$ entre os meninos e de $12,7 \%$ a $18,6 \%$ entre as meninas. Em Portugal ${ }^{(14)}, 17,6 \%$ da amostra estudada apresentou hipercolesterolemia. No Paquistão(15) verificou-se prevalência alarmante de colesterol total aumentado na população infantil: 62\% entre as meninas e 54\% entre os meninos. A partir destes dados, verificamos que a prevalência encontrada $(4,7 \%)$ no atual estudo pode ser considerada baixa.

Do total da amostra foram encontradas 8,9\% das crianças com hipertrigliceridemia, assemelhando-se aos estudos realizados nos município de Itajaí-SC ${ }^{(4)}(4,7 \%)$ e Campina Grande-PB(12) $(11,1 \%)$.

A prevalência de excesso de peso do presente estudo (20,7\%) mostrou-se superior às encontradas em Florianópolis ${ }^{(16)}(15,6 \%)$, Recife-PE $E^{(17)}$ $(14,7 \%),(10,8 \%)$, Campina Grande-PB(12) $(14,4 \%),(13,7 \%)$, e em cidades das regiões Nordeste e Sudeste ${ }^{(18)}(17,3 \%)$. No entanto, mostrou-se inferior as prevalências encontradas em estudos realizados em Santos-SP(19) $(33,7 \%)$ e no Nordeste brasileiro $(33,6 \%)^{(20)}$.

Não há ainda consenso na literatura em relação às diferenças estatísticas entre os sexos para as variáveis analisadas. Franca e Alves(21), Leão et al. ${ }^{(22)}$ e Abrantes et al. ${ }^{(18)}$ encontraram prevalências superiores de excesso de peso para o sexo feminino. Soar et al. ${ }^{(23)}$, Chu e Pan ${ }^{(24)}$ e Silva et al. ${ }^{(16)}$, relataram percentuais superiores no sexo masculino. No presente estudo não foi encontrada diferença significante entre os sexos, como também relatado em outros trabalhos ${ }^{(20,25)}$.

Diferentemente de outros estudos ${ }^{(4,16,19)}$, este trabalho não encontrou diferença estatística significante entre a rede privada e pública nas variáveis estudadas (tabelas 1 e 3). Possivelmente, esta ausência de diferença entre as redes de ensino ocorreu em função: a) do caráter filantrópico de algumas instituições particulares que participaram do trabalho. Duas das cinco escolas privadas estão localizadas em áreas urbanas consideradas de baixa renda. Estas são mantidas por redes filantrópicas que possuem renda oriunda de escolas localizadas em áreas nobres; b) a distribuição da população de alta renda. Muitas dessas famílias moram na periferia, onde, geralmente, a escola mais acessível para as crianças é uma escola pública; e c) transição nutricional(26-28). o aumento do sedentarismo e maior disponibilidade de alimentos, 
anteriormente relatados apenas em altos níveis socioeconômicos, que atualmente está atingindo todas as classes sociais, consequente ao padrão de vida moderna.

Na amostra estudada, grande número de crianças (14,1\% a 19,4\% da amostra) encontra-se na faixa limítrofe para CT ou apresentam sobrepeso. Tais resultados evidenciam a importância da atenção, orientação e prevenção a cerca do excesso de peso e alterações lipídicas cada vez mais precoces na população pediátrica(29). A obesidade hoje não se resume a um problema de países desenvolvidos, mas sim, afeta cada vez mais países menos favorecidos ${ }^{(27)}$.

A estratificação da amostra em CT limítrofe e aumentado, conforme estado nutricional (tabela 4) permitiu observar que as maiores prevalências de CT limítrofe e alterado encontram-se entre as crianças obesas, seguidas das com sobrepeso. Das 19 crianças obesas, apenas oito (42,1\%) apresentaram valores para CT ideais. Estes achados condizem com os relatados em Taiwan ${ }^{(24)}$, onde os autores avaliaram a prevalência de obesidade e as comorbidades associadas em 2.405 crianças e verificaram que a prevalência das comorbidades relacionadas à obesidade mostram-se significantemente maiores entre os escolares com obesidade ou sobrepeso. Isto nos permite consolidar a ideia de que a obesidade é um agravo nutricional que se associa a uma alta incidência de doenças cardiovasculares, câncer, diabetes, síndrome metabólica e dislipidemias, influenciando, desta maneira, no perfil de morbimortalidade das populações ${ }^{(27,30)}$.

\section{REFERÊNCIAS}

1. Nieman DC. Exercício e saúde: como se prevenir de doenças usando o exercício como seu medicamento. São Paulo: Manole; 1999

2. Moura EC, de Castro CM, Mellin AS e Bueno D. Perfil lipídico em escolares de Campinas, SP, Brasil. Rev Saúde Pública 2000;34:499-505.

3. Gotto AM Jr. Triglyceride as a risk factor for coronary artery disease. Am J Cardiol 1998;82:22Q-25Q.

4. Gerber ZR, Zielinski P. Fatores de risco de aterosclerose na infância. Um estudo epidemiológico. Arq Bras Cardiol 1997;64:231-6.

5. Committee on Nutrition. Cholesterol in childhood. Pediatrics 1998; 101

6. Brotons C, Ribera A, Perich RM, Abrodos D, Magana P, Pablo S, et al. Worldwide distribution of blood lipids and lipoproteins in childhood and adolescence: a review study. Atherosclerosis 1998;139:1-9.

7. Coronelli CL, Moura EC. Hipercolesterolemia em escolares e seus fatores de risco. Rev Saúde Publ 2003;37: 24-31

8. Alvarez BR, Pavan AL. Alturas e Comprimentos. In: Petroski EL, editor. Antropometria: técnicas e padronizações. Porto Alegre: Palotti; 2003. p. 31-45

9. Cole TJ, Bellizzi MC, Flegal KM, Dietz WH. Establishing a standard definition for child overweight and obesity worldwide: international survey. BMJ 2000;320:1240-3.

10. Santos RD, Maranhão RC, Luz PL, Lima JC, Filho WS, Avezum A, et al. III Diretrizes brasileiras sobre dislipidemias e diretrizes de prevenção da aterosclerose do Departamento de Aterosclerose da Sociedade Brasileira de Cardiologia. Arq Bras Cardiol 2001;77:1-191

11. Grillo LP, Crispim SP, Siebert AN, et al. Perfil lipídico e obesidade em escolares de baixa renda. Rev Bras Epidemiol 2005;8:75-81.

12. Carvalho DF, Paiva AA, Melo ASO, Ramos AT, Medeiros JS, et al. Perfil lipídico e estado nutricional de adolescentes. Rev Bras Epidemiol 2007;10:491-8.

13. Webber LS, Osganian V, Suepker RV, Feldman HA, Stone EJ, Elder JP, et al. Cardiovascular risk factors among third grade children in four regions of the United States. Am J Epidemiol 1995;141:428-39.

14. Santiago LM, Sá O, de Carvalho IM, Rocha MG, Palmeiro L, Mesquita EP, et al. Hipercolesterolemia e factores de risco cardiovascular associados, em crianças e adolescentes. Rev Port Cardiol 2002;21:301-13.

15. Badruddin SH, Khurshid M, Molla A, Manser WW, Lalani R, Vellani CW. Factors Associated with elevated serum cholesterol levels in well-to-do Pakistani scholchildren. J Trop Med Hyg 1991;94:123-9.

16. Silva KS, Pelegrini A, Hoefelmann LP, Vasques DG, Lopes, AS. Prevalência de excesso de peso cor-
Há relatos de diminuição das prevalências de obesidade na Escandinávia. Especula-se que este fato se deva ao intenso trabalho da mídia ao combate ao estilo de vida sedentário e promoção de hábitos alimentares saudáveis ${ }^{(26)}$. Desta forma, reforça-se a importância de políticas públicas e a necessidade de assistência pediátrica nessa faixa etária, visando o seu diagnóstico precoce e, principalmente, o aconselhamento nutricional e incentivo à prática esportiva, uma vez que as dislipidemias e o excesso de peso têm sido apontados como fatores de risco para as doenças cardiovasculares.

A prevalência de excesso de peso dos estudantes de Santa Maria mostrou-se relativamente alta, já a prevalência de alterações lipídicas pode ser considerada baixa. Não foi encontrada diferença significante entre sexos e rede particular e pública na amostra estudada.

\section{AGRADECIMENTOS}

Os autores agradecem à Dirce dos Santos Xavier e à Maria de Lourdes Cruz Lunardi por realizarem as coletas de dados sempre com grande dedicação e entusiasmo, e às escolas e crianças participantes que se disponibilizaram para a realização deste estudo.

Todos os autores declararam não haver qualquer potencial conflito de interesses referente a este artigo. poral em escolas publicas e privadas da cidade de Florianópolis, SC. Arq Bras Endocrinol Metab 2008;52:574-5.

17. Motta MEFA, Silva GAP. Desnutrição e obesidade em crianças: delineamento do perfil de uma comunidade de baixa renda. J Pediatr 2001;77:288-93.

18. Abrantes MM, Lamounier JA, Colosimo EA. Prevalência de sobrepeso e obesidade nas regiões Nordeste e Sudeste do Brasil. Rev Assoc Med Bras 2003;49:162-6.

19. Costa RF, Cintra IP, Fisberg M. Prevalência de sobrepeso e obesidade em escolares da cidade de Santos, SP. Arq Bras Endocrinol Metab 2006;50:60-7.

20. Brasil LMP, Fisberg M e Marrano HS. Excesso de peso de escolares em região do Nordeste Brasileiro: contraste entre as redes de ensino pública e privada. Rev Bras Saúde Matern Infant 2007;7:405-12.

21. Franca E, Alves, JG. Dislipidemia entre crianças e adolescentes de Pernambuco. Arq Bras Cardiol 2006;87:722-7.

22. Leão LSCS, Araújo LMB, Moraes LTLP, Assis AM. Prevalência de obesidade em escolares de Salvador, Bahia. Arq Bras Endocrinol Metab 2003;47:151-7.

23. Soar C, Vasconcelos FAG, Assis MAA, Grosseman S e Luna MEP. Prevalência de sobrepeso e obesidade em escolares de uma escola pública de Florianópolis, Santa Catarina. Rev Bras Saúde Matern Infant 2004;4:391-7.

24. Chu NF, Pan WH. Prevalence of obesity and its comorbidities among schoolchildren in Taiwan. Asia Pac J Clin Nutr 2007;16:601-7.

25. Oliveira AMA, Cerqueira EMM, Oliveira AC. Prevalência de sobrepeso e obesidade infantil na cidade de Feira de Santana, Bahia: detecção na família x diagnóstico clínico. J Pediatr 2003;79:325-8.

26. Monteiro CA, Benicio MH, Conde WL, Pookin BM. Shifting obesity trends in Brazil. Eur J Clin Nutr 2000;54:342-6

27. Kac G, Velasquez-Melendez G. A transição nutricional e a epidemiologia da obesidade na America Latina. Cad Saúde Publica 2003;19:54-5.

28. Batista Filho M, Rissin A. A transição nutricional no Brasil: tendências regionais e temporais. Cad Saúde Pública 2003;19:S181-91.

29. Giuliano ICB, Caramelli B, Pellanda L, Duncan B, Mattos S, Fonseca FH. I diretriz de prevenção da aterosclerose na infância e na adolescência. Arq Bras Cardiol 2005;85.

30. Gus M, Fuchs SC, Moreira LB, Moraes RS, Wiehe M, Silva AF, et al. Association between different measurements of obesity and the incidence of hypertension. Am J Hypertens 2004;17:50-3. 